$\begin{array}{llll}\Omega & \square & \equiv & \text { Q } \\ \text { A. Aujayeb', } & \text { Royal Victoria Infirmary, } & \text { Royal Victoria Infirmary, } & \text { aujayeb@doctors.net.uk } \\ \text { S. Worthy', } & \text { Newcastle Upon Tyne } & \text { Newcastle Upon Tyne } & \\ \text { C. Stenton' } & \text { Hospitals, Newcastle upon } & \text { Hospitals, Queen Victoria } & \\ & \text { Tyne, UK } & \text { Road, Newcastle upon Tyne, } & \\ & & \text { NEx 4LP, UK } & \end{array}$

\title{
Black lungs and tuberculosis
}

\section{Case report}

A never-smoking 82-year-old woman with a past medical history of type-2 diabetes presented to the outpatient clinic with a 34 months history of purulent bronchitis, haemoptysis, weight loss and fevers.

She had lived in a small village in India and Pakistan all her life and had been visiting the UK intermittently until setting up permanent residency a few months ago.

She denied any history of tuberculosis or any previous recent contacts.

Clinically, she had no lymphadenopathy and had bilateral crackles in the upper zones on auscultation. Her oxygen saturations were $98 \%$ on air.

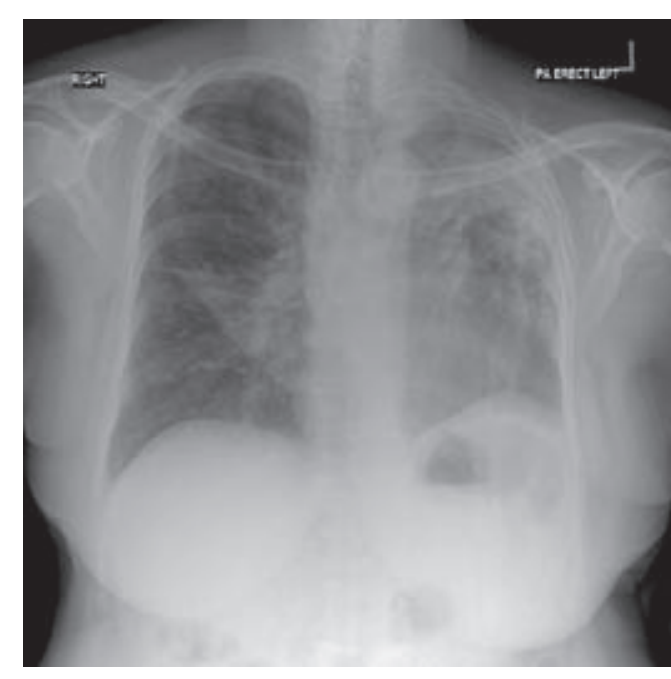

Figure 1

Chest radiograph

\section{Task 1}

Interpret the chest radiograph (fig. 1) 
Answer 1. The chest radiograph shows generalised nodularity with left upper lobe collapse and right middle lobe collapse. Crowding of the left upper ribs suggests long-standing volume loss.

A diagnosis of tuberculosis was entertained and sputum sent for analysis. A helical CT scan was also performed.

\section{Task 2}

Please interpret her CT scans (fig. 2)
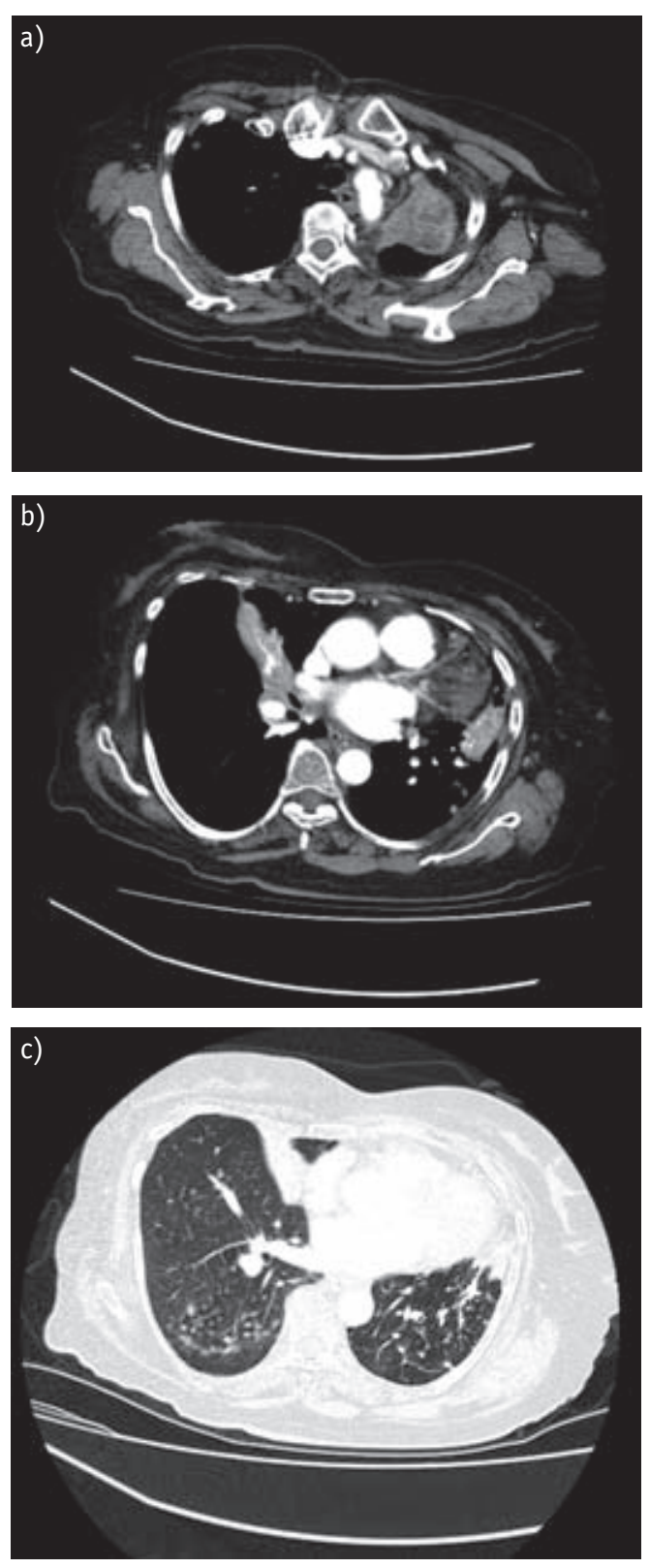

Figure 2

Computerised tomography images 
Answer 2. There is right middle lobe collapse with fluid-filled distal bronchi and patent origin of bronchus. There is marked volume loss and scarring in left upper lobe with fluid-filled bronchi and distorted left upper lobe bronchus, but also a larger low attenuation area on the left peri-hilar region. There are also numerous mainly peri-bronchovascular nodules in both lungs. There is also reduced lung attenuation in left lower lobe

Her sputum cultures yielded no positive results and hence she underwent fibre optic
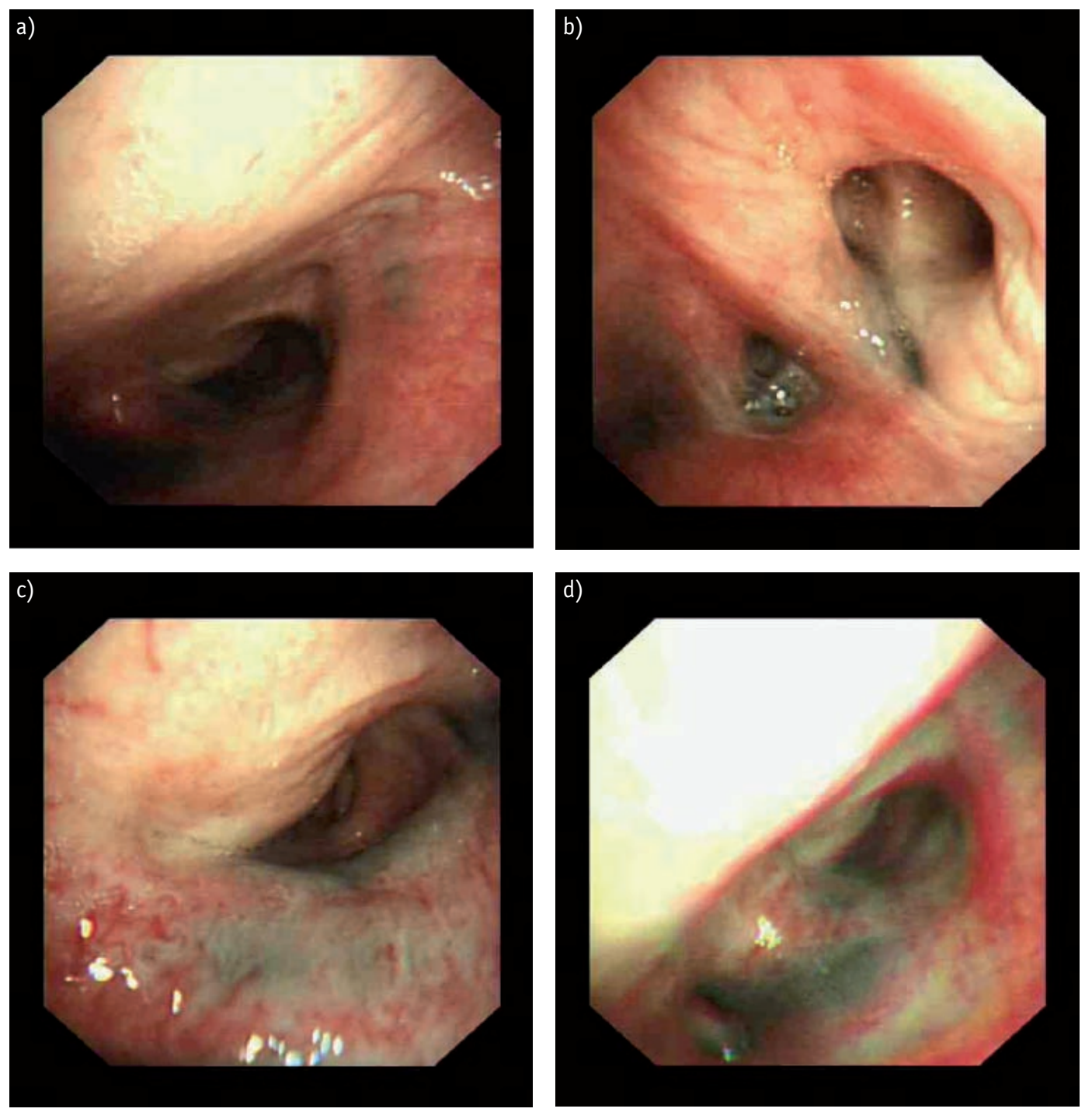

bronchoscopy with the view of obtaining lavages for culture and microscopy.

Figure 3

Bronchoscopy images

\section{Task 3}

Interpret the bronchoscopic images

(fig. 3) 
Answer 3. There are areas of black pigmentation, which is known as anthracosis along with bronchial wall narrowing. Histology would classically show soot-laden macrophages.

She was started on anti-tuberculous chemotherapy consisting of pyrazinamide, ethambutol, rifampicin and isoniazid given the high index of suspicion. She had a reaction to the medications which included vomiting and a transient bilirubin rise, which recurred when single agents were slowly reintroduced. No acid fast bacilli (AFB) were seen on direct microscopy of the bronchial lavage and cultures were negative.

On repeated follow-up visits, she felt very well with no reported symptoms or ongoing weight loss. Even if she had active tuberculosis, she was non-infective. After a discussion with the patient, her family and all health practitioners about the risk-benefits of treatment versus non-treatment and careful followup, a decision was reached to not start any further treatment.

A more detailed history revealed that she had been a housewife all her life in a rural region of India and Pakistan and had had no exposure to coal. However, she has cooked on an open wood fire all her life prior to coming to the UK.

With the CT and bronchoscopic appearances, we conclude that the diagnosis was of bronchial anthracofibrosis (BAF), post-tuberculous scarring and possible superimposed active tuberculosis.

\section{Discussion}

Bronchial anthracofibrosis (BAF) is a term that encompasses bronchial wall narrowing and anthracotic pigmentation visible on the bronchial walls. The term was first used in a case series from Korea [1] where the predominance seemed to be in never-smoking elderly female subjects with prolonged sustained exposure to wood smoke. The narrowing and pigmentation was mostly seen in the right middle lobe. 17 of the 28 patients in that case series also had active pulmonary tuberculosis. However, endobronchial pigmentation with airway narrowing was first described in 1951 by ABRAHAM [2] in patients with perforated tuberculous lymph nodes.

In adults who are exposed to carbon materials, mild lung deposition is common and benign. In susceptible individuals with overexposure in various occupations, such as coal mining, may result in significant parenchymal disease such as coal workers' pneumoconiosis [3]. BAF has been postulated to be caused by endobronchial tuberculosis [4]. Endobronchial TB or tuberculous lymphadenopathy are more common in young women, hence perforation of a lymph node can occur very slowly with no clinical symptoms and the healing process would manifest as fibrosis and be discovered later in life. However, it has been observed that BAF is not synonymous with active or old tuberculosis. A series of 102 patients from Iran [5] confirmed BAF in full-time housewives living in rural regions and using biomass as fuel but none of them had tuberculosis. A series of patients from the UK had occupational exposure to coal dust and only one had past history of tuberculosis [6]. Alternatively, BAF has been thought to be an endobronchial

\section{Key learning points}

- Anthracosis can occur without coal exposure and is more common in elderly women who have been exposed to biomass fuel pollution.

- Anthracofibrosis is a clinical entity where there is anthracosis and bronchial wall stenosis. This predominantly affects the right middle lobe.

- There is an association of anthracofibrosis with tuberculosis.

- Typical CT appearances are distal atelectasis and lymphadenopathy and the main differential diagnosis is of malignancy.

- Bacteriological evidence of TB should be ascertained before starting anti-tuberculous chemotherapy. 
form of pneumoconiosis and a variant of progressive massive fibrosis and the term "hut lung" was coined to describe a form of pneumoconiosis in rural African women [7].

In a survey of 260,000 Indian adults [8] and in a case-control study [9], tuberculosis was more common in people exposed to biomass fuel pollution. Chronic smoke exposure might impair the mucociliary defences of the respiratory system and reduce macrophage activity, increasing susceptibility to even mycobacterial infections [10]. On the other hand, HWANG et al. [11] found that carbonaceous particles, silica and silicates predominated in tissue specimens of patients with BAF, which might explain the predisposition to tuberculosis.

Radiological features of BAF have been described. In a case series from Korea [12], $94 \%$ of patients had atelectasis distal to the smoothly narrowed bronchus. $94 \%$ of patients also had mediastinal or hilar lymphadenopathy and $57 \%$ of those were calcified. The main differential diagnosis is of malignancy and careful evaluation of the patients should be undertaken. Parenchymal abnormalities can include consolidation, low attenuation nodules and interstitial infiltrations. Various reasons have been postulated for the predisposition of BAF for the right middle lobe; namely its location in relation to the bronchus intermedius or anatomical and ineffective collateral ventilation [13].

It had been postulated that anti-tuberculous chemotherapy should always be given to someone with BAF but current evidence demands positivity of cultures first [14]. We started the quadruple regimen as the patient had reported systemic symptoms. On followup, she had remained stable clinically and radiologically. With non-tuberculous BAF, steroids achieved clinical improvement but with post tuberculous BAF, there is no consensus on treatment [15].

Our patient has remained well on follow up with no change in her radiological appearance and clinical state.

References

1. Chung MP, Lee KS, Han J, et al. Bronchial stenosis due to anthracofibrosis. Chest 1998; 113: 344-350.

2. Abraham GC. Atelectasis of the right middle lobe resulting from perforation of tuberculous lymph nodes into bronchi in adults. Ann Intern Med 1951; 35: $820-835$.

3. Alois D. Pneumoconioses: Encyclopedia of occupational health and safety, Geneva: International Labour Organization, 1998; p. 10.32.

4. Kim JY, Park JS, Kang MJ, et al. Endobronchial anthracofibrosis is causally associated with tuberculosis. Korean J Intern Med 1996; 51: 351-357.

5. Amoli K. Bronchopulmonary disease in Iranian housewives chronically exposed to indoor smoke. Eur Respir J 1998; 11: 659-663.

6. Wynn G), Turkington PM, O'Driscoll BR. Anthracofibrosis, Bronchial Stenosis With Overlying Anthracotic Mucosa: Possibly a New Occupational Lung Disorder* A Series of Seven Cases From One UK Hospital. Chest 2008; 134: 1069-1073.

7. Grobbelaar JP, Bateman ED. Hut lung: a domestically acquired pneumoconiosis of mixed aetiology in rural women. Thorax. 1991; 46: 334-340.

8. Mishra VK, Retherford RD, Smith KR. Biomass cooking fuels and prevalence of tuberculosis in India. Int J Infect Dis 1999; 3: 119-129.
9. Pérez-Padilla R, Pérez-Guzmán C, Báez-Saldaña R, et al. Cooking with biomass stoves and tuberculosis: a case-control study. Int J Tuberc Lung Dis 2001; 5: $441-447$.

10. Smith KR, Samet JM, Romieu I, et al. Indoor air pollution in developing countries and acute lower respiratory infections in children. Thorax 2000; 55: 518-532.

11. Hwang J, Puttagunta L, Green F et al. Bronchial anthracofibrosis and tuberculosis in immigrants to Canada from the Indian subcontinent. Int J Tuberc Lung Dis 2010; 14: 231-237.

12. Kim HY, Im JG, Goo JM, et al. Bronchial anthracofibrosis inflammatory bronchial stenosis with anthracotic pigmentation: CT findings. Am J Roentgenol 2000; 174: 523-527.

13. Brock RC. Post-tuberculous broncho-stenosis and bronchiectasis of the middle lobe. Thorax 1950; 5: 539.

14. Han SH, Cha GY, Lee YM, et al. Study of antituberculous medications in antracofibrosis. Tuberc Respir Dis 2001; 51: 224-231.

15. Jang SJ, Lee SY, Kim SC, et al. Clinical and radiological characteristics of non-tuberculous bronchial anthracofibrosis. Tuberc Respir Dis 2007; 63: 139-144. 